\title{
NULL-ORBIT REFLEXIVE OPERATORS
}

\author{
Don Hadwin, ILEANa IONAŞCU AND HaSSAN YOUSEFI
}

\begin{abstract}
We introduce and study the notion of null-orbit reflexivity, which is a slight perturbation of the notion of orbit-reflexivity. Positive results for orbit reflexivity and the recent notion of $\mathbb{C}$-orbit reflexivity both extend to null-orbit reflexivity. Of the two known examples of operators that are not orbit-reflexive, one is null-orbit reflexive and the other is not. The class of null-orbit reflexive operators includes the classes of hyponormal, algebraic, compact, strictly block-upper (lower) triangular operators, and operators whose spectral radius is not 1 . We also prove that every polynomially bounded operator on a Hilbert space is both orbit-reflexive and null-orbit reflexive.
\end{abstract}

Mathematics subject classification (2010): 47A15.

Keywords and phrases: Operators, subspaces, null-orbit reflexivity.

\section{REFERENCES}

[1] Sophie Grivaux, Maria Roginskaya, On Read's type operators on Hilbert spaces, Int. Math. Res. Not. IMRN 2008, Art. ID rnn 083, 42 pp.

[2] Don Hadwin, Algebraically reflexive linear transformations, Linear and Multilinear Algebra 14 (1983), 225-233.

[3] Don Hadwin, Ileana Ionaşcu, Michael McHugh, Hassan Yousefi, $\mathbb{C}$-orbit reflexive operators, Operators and Matrices, to appear (arXiv:1005.5202).

[4] Don Hadwin, Eric Nordgren, Reflexivity and direct sums, Acta Sci. Math. (1991), 181-197.

[5] Don Hadwin, Eric Nordgren, Heydar Radjavi, Peter Rosenthal, Orbit-reflexive operators, J. London Math. Soc. (2) 34, 1 (1986), 111-119.

[6] IRVING KAPLANSKY, Infinite abelian groups, Revised edition, The University of Michigan Press, Ann Arbor, Mich. 1969ssertation, University of New Hampshire, 1995.

[7] DaVID R. LARSON, Reflexivity, algebraic reflexivity and linear interpolation, Amer. J. Math. 110 (1988), 283-299.

[8] LeOnya Livshits, Locally finite-dimensional sets of operators, Proc. Amer. Math. Soc. 119, 1 (1993), 165-169.

[9] W. MLAK, Operator valued representations of function algebras. Linear operators and approximation. II, (Proc. Conf., Oberwolfach Res. Inst., Oberwolfach, 1974), Internat. Se. Numer. Math., Vol. 25, Birkhäuser (Basel, 1974).

[10] Vladimír MüLler, Kaplansky's theorem and Banach PI-algebras, Pacific J. Math. 141, 2 (1990), 355-361.

[11] V. MÜLLER, J. VRŠOVSKÝ, On orbit-reflexive operators, J. Lond. Math. Soc. (2) 79, 2 (2009), 497 510. 
Operators and Matrices www. ele-math.com 